\section{CVIA}

CASE REPORT

pISSN 2508-707X / eISSN 2508-7088 https://doi.org/10.22468/cvia.2018.00283 CVIA 2019;3(3):93-95

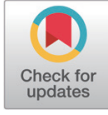

\title{
Role of Cardiac MRI in Complicated Cardiac Defects
}

\author{
Yasir Khan, Mohammad Bin Pervez, Saulat Fatimi \\ Section of Cardiothoracic, Department of Surgery, Aga Khan University Hospital, \\ Karachi, Pakistan
}

Received: December 5, 2018

Revised: May 10, 2019

Accepted: June 7, 2019

Corresponding author

Yasir Khan, MBBS

Section of Cardiothoracic,

Department of Surgery,

Aga Khan University Hospital,

Stadium Road, PO Box 3500,

Karachi 74800, Pakistan

Tel: 92-21-3486-4708

Fax: 92-21-3493-2095

E-mail: yasir.bilal@aku.edu

\begin{abstract}
Sinus of Valsalva rupture is a rare condition that results in aortocardiac shunt. Although the investigation of choice is transthoracic echocardiography, the gold standard is cardiac catheterization. We present a case of a young male with acute onset heart failure. Acute coronary syndrome blood workup was unremarkable. Transthoracic and transesophageal echocardiography revealed mild right atrial dilation with a Gerbode shunt. Right heart catheterization showed left to right shunt suggestive of acquired acute onset shunting. Eventually, a cardiac MRI revealed a ruptured sinus of Valsalva opening into the right atrium as the cause. The patient was taken to the operation theater where an aortobicaval bypass was established. A horizontal aortotomy revealed a sinus of Valsalva aneurysm arising from the right coronary sinus which was repaired using a Dacron patch. Postoperative transthoracic echocardiography showed no defect. In summary, cardiac MRI is an excellent tool for establishing a definite diagnosis for complicated cardiac defects.
\end{abstract}

Key words Ruptured aortic aneurysm · Congenital defects · Magnetic resonance imaging, functional $\cdot$ Cine magnetic resonance imaging.

\section{INTRODUCTION}

Sinus of Valsalva aneurysms (SVA) are rare, occurring in $0.15 \%$ to $1.5 \%$ of patients. They can either be congenital or acquired [1]. Congenital aneurysms may result from localized weakness of the elastic lamina or an underlying deficiency of normal elastic tissue. Acquired aneurysms are commonly caused by infectious diseases such as bacterial endocarditis, syphilis, and tuberculosis, degenerative conditions such as atherosclerosis and cystic medial necrosis, and injury from deceleration trauma [2]. Most Valsalva sinus aneurysms are seen at echocardiography, but the gold standard diagnostic method is cardiac catheterization and aortography [3]. However, recent studies showed the importance of cardiac MRI in a truly comprehensive assessment of cardiac anatomy, function and flow, making it well suited for SVA assessment [4]. Here, we present a case of ruptured sinus of Valsalva aneurysm that was missed by echocardiography but confirmed through cardiac magnetic resonance imaging.

(c) This is an Open Access article distributed under the terms of the Creative Commons Attribution Non-Commercial License (https://creativecommons.org/licenses/bync/4.0) which permits unrestricted non-commercial use, distribution, and reproduction in any medium, provided the original work is properly cited.

\section{CASE REPORT}

A 40-year-old male presented with symptoms of heart failure in the form of severe dyspnea. He had no risk factors for coronary artery disease and no family history of congenital heart disease. On examination, he had a pansystolic murmur. Initial investigations showed pulmonary edema on the chest X-ray. The acute coronary syndrome blood workup was unremarkable. Transthoracic echocardiography showed mild right atrium and right ventricle dilation with turbulence just below the tricuspid valve, suggestive of a Gerbode type ventriculoatrial defect (Fig. 1C). Right ventricular pressures were greater than half systemic pressure along with moderate pulmonary hypertension. Transesophageal echocardiography conducted for further delineation also suggested a Gerbode shunt. Right heart catheterization was performed and showed elevated right-sided filling pressure with a right atrium pressure of about $33 \mathrm{~mm} \mathrm{Hg}$, step up in the right atrium, and right ventricle and pulmonary artery with $55 \%$ saturation in the superior vena cava and $82 \%$ in the right atrium, Qp:Qs of 3:1 but with normal pulmonary resistance of $2.4 \mathrm{WU}$ and pulmonary vascular resitance/systemic vascular resistance of 0.08 . Overall, the findings of the right heart catheterization were suggestive of left-to-right shunt of acute onset, which made the diagnosis of a Gerbode defect doubtful. 

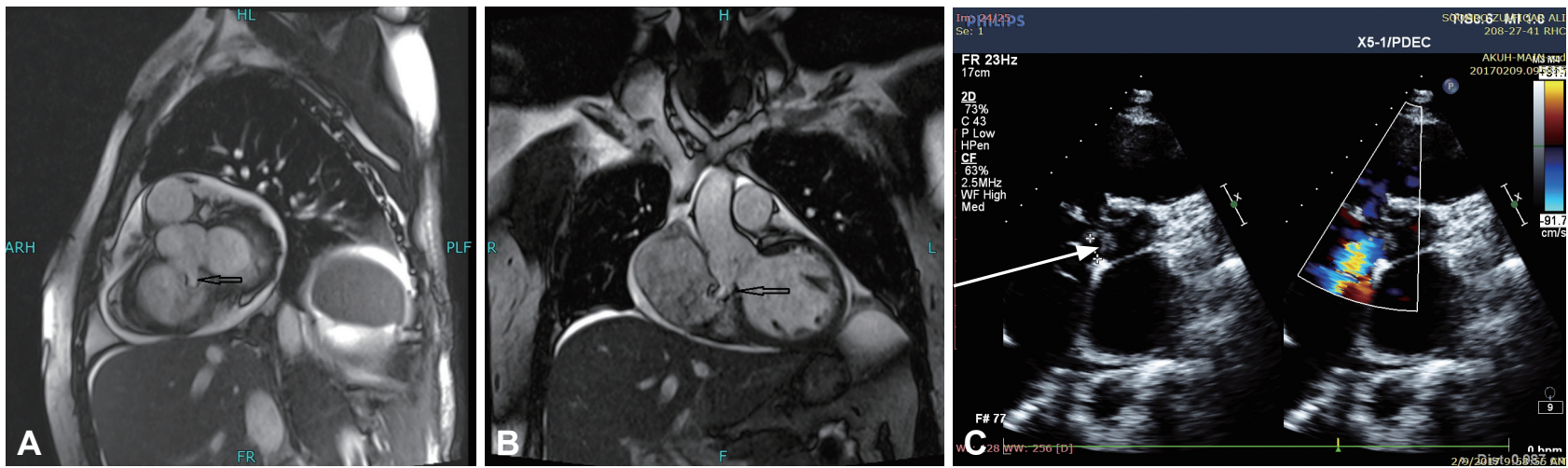

Fig. 1. Imaging showing intra cardiac defect. A: Cardiac MRI short axis cine sequence showed sinus of Valsalva aneurysm that ruptured into the right atrium (arrow). B: Coronal Cardiac MRI cine sequence showed sinus of Valsalva aneurysm that ruptured into the right atrium (arrow). C: Echocardiographic image suggested a Gerbode defect (arrow).

Eventually, cardiac MRI was performed. Cardiac MRI instead of electrocardiographic (ECG) gated CT was performed because cardiac MRI was thought to be a better modality to look for left ventricular hemodynamics, fistulous blood flow, valvular pathology, magnitude of aortocardiac shunt, and to identify the exact pathology of the aortocardiac shunt (Supplementary Video 1 in the online-only Data Supplement). Accordingly, the cardiac MRI showed a ruptured sinus of Valsalva arising from the right coronary sinus and opening into the right atrium as the offending cause (Fig. 1A and B). The patient was taken to the operation theater where aortobicaval bypass was established. A horizontal aortotomy revealed a SVA arising from the right coronary sinus which was repaired using a Dacron patch (Fig. 2). A postoperative transthoracic echocardiography showed no defect. The patient remained well at his last clinic visit at 10 months postoperative. Informed consent has been taken from the patient.

\section{DISCUSSION}

SVA rupture and the Gerbode defect are two different and complex cardiac defects requiring different surgical approaches for repair. A ruptured SVA is an uncommon condition with variable manifestations that result in aortocadiac shunt [3]. SVA can either be congenital or acquired. Depending on the size of the aneurysm, the rapidity with which it ruptures and the cardiac chamber with which it communicates vary. The symptoms of a ruptured SVA may include severe dyspnea, chest pain, and hemodynamic compromise, or insidiously progressive heart failure with fatigue, dyspnea, and volume overload [3]. Aneurysm rupture most commonly occurs in the third or fourth decade of life, and it may be spontaneous or precipitated by trauma or strenuous physical exertion. Most right coronary sinus aneurysms rupture into the right ventricular outflow tract, although the pulmonary artery and interventricular septum may sometimes be involved. Aneurysms of the noncoronary sinus usually rupture into the right atrium. Aneurysms of the left coronary sinus

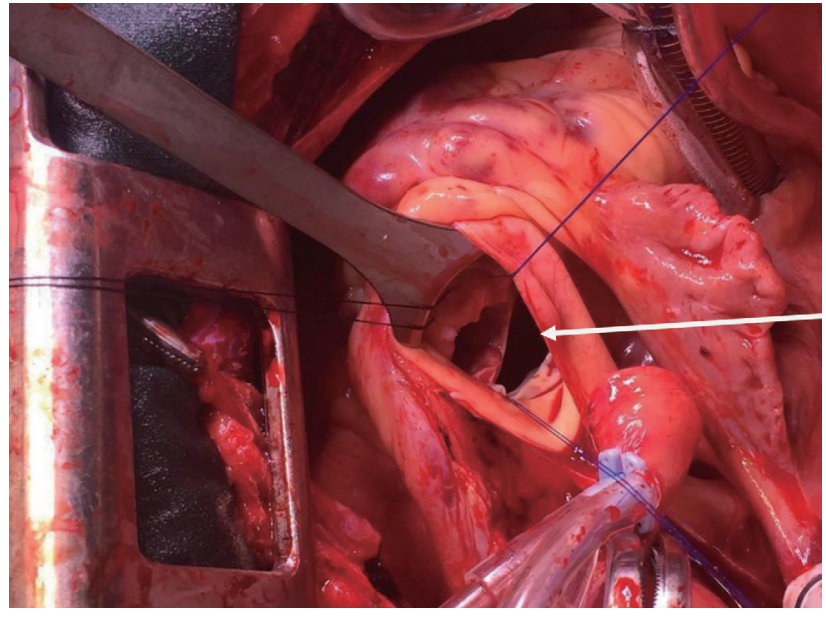

Fig. 2. An intraoperative image demonstrated a ruptured sinus of Valsalva. The arrow indicates the ruptured sinus of Valsalva.

may rupture into the left ventricle or left atrium [2]. The goldstandard diagnostic method for SVA is cardiac catheterization and aortography [3]. Initial diagnoses of SVAs are established by two-dimensional and Doppler color echocardiography, but some cases missed on echocardiography were discovered on cardiac MRI and cardiac CT in one report [3]. The advantages of cardiac MRI over ECG-gated CT scan include the ability to evaluate the left ventricular hemodynamic pattern, identify aortic regurgitation, and quantify any aortocardiac shunt or turbulent or fistulous blood flow [4]. Ruptured Valsalva sinus aneurysms are associated with potentially fatal complications. However, the prognosis after treatment is excellent [2]. The treatment of ruptured Valsalva sinus aneurysm is surgery.

Gerbode defect is classified into two types: direct and indirect. Direct defects transcend the membranous septum from the left ventricular to the right atrium, while indirect defects involve a ventricular septal defect with accompanying tricuspid regurgitation. This classification was later modified to describe the position of the anomaly in relation to the tricuspid valve. Approximately one-third of such defects occurs in the atrioven- 
tricular septum and is known as supravalvular defects. The remaining two-thirds occur between the ventricles and are known as infravalvular defects. The initial diagnostic examination of choice is transthoracic echocardiography, but color flow Doppler cardiac MRI can reveal further detailed anatomical and physiological information. Cardiac MRI can enhance the shunt anatomy, measure left and right heart volumes, and quantify shunt flow [5]. Treatment of a Gerbode defect is surgical closure.

Cardiac MRI has emerged as an excellent noninvasive modality; it can provide a truly comprehensive assessment of cardiac anatomy, function and flow, making it well suited for SVA assessment [3]. Recent developments in scanner technology, including parallel imaging, respiratory gating, and arrhythmia rejection capabilities, can now yield diagnostic quality images in patients for whom cardiac MRI would previously have been futile [6].

In summary, for complicated cardiac defects, like rupture of the sinus of Valsalva, that require prompt and definite diagnosis, Cardiac MRI is an excellent alternative tool to establish a definite diagnosis. This is particularly important in planning the operative approach. If the surgeon is aware of the exact nature of the defect, he can better plan his intervention for a disease process that often requires creative solutions.

\section{Supplementary Video Legends}

Video 1. Video showing rupture sinus of Valsalva into right atrium.

\section{Supplementary Materials}

The online-only Data Supplement is available with this article at https:// doi.org/10.22468/cvia.2018.00283.

\section{Conflicts of Interest}

The authors have no potential conflicts of interest to disclose.

\section{REFERENCES}

1. Fathala A. Depiction of ruptured sinus of Valsalva aneurysms by cardiac computed tomography angiography. Exp Clin Cardiol 2012;17:152-154.

2. Hoey ET, Kanagasingam A, Sivananthan MU. Sinus of Valsalva aneurysms: assessment with cardiovascular MRI. AJR Am J Roentgenol 2010; 194:W495-W504.

3. Moustafa S, Mookadam F, Cooper L, Adam G, Zehr K, Stulak J, et al. Sinus of Valsalva aneurysms-- 47 years of a single center experience and systematic overview of published reports. Am J Cardiol 2007;99:1159-1164.

4. Bricker AO, Avutu B, Mohammed TL, Williamson EE, Syed IS, Julsrud $\mathrm{PR}$, et al. Valsalva sinus aneurysms: findings at $\mathrm{CT}$ and MR imaging. Radiographics 2010;30:99-110.

5. Saker E, Bahri GN, Montalbano MJ, Johal J, Graham RA, Tardieu GG, et al. Gerbode defect: a comprehensive review of its history, anatomy, embryology, pathophysiology, diagnosis, and treatment. J Saudi Heart Assoc 2017;29:283-292.

6. Hoey ET, Gulati GS, Singh S, Watkin RW, Nazir S, Ganeshan A, et al. The role of multi-modality imaging for sinus of Valsalva aneurysms. Int J Cardiovasc Imaging 2012;28:1725-1738. 